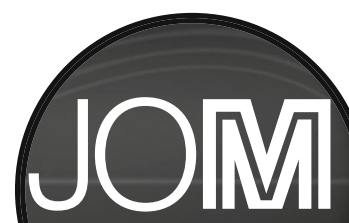

\title{
2014 editorial calendar
}

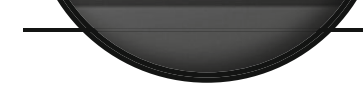

\section{August 2014}

Theme: Physical Metallurgy

- Aluminum: Shaping and Forming

- Microstructural Patterning during Phase Transformations

- Semisolid Deformation: Experimental and Numerical Developments

- Multiscale Approaches to Hydrogen-Assisted Degradation of Metals

- Heterogenous Nucleation and Initial Microstructural Formation

\section{Manuscripts Due: Deadline Passed}

\section{September 2014}

\section{Theme: Metals and Alloys: Part II}

- Energy Savings in Mining and Metallurgical Industries

- Critical Metals Hydrometallurgy

- Modeling, Simulation and Development of Metallurgical Processes

- Refractory Metals and Alloys

- Low-Density Steels

Manuscripts Due: Deadline Passed

\section{October 2014}

Theme: Mechanical Properties

- Progress in High-Entropy Alloys

- Multi-Objective Optimization for Materials

- Multiscale Perspective of Interface-related Mechanics of Nanocomposites

- Deformation and Forming of Joined Materials

- Multi-scale Modeling: Concurrent and Hierarchical Methods

Manuscripts Due: Deadline Passed

\section{November 2014 \\ Theme: Energy and Environment}

- Aluminum: Recycling and Environmental Issues

- Alloys and Compounds for Thermoelectric and Solar Cell Applications

- Progress with Lead-free Solders

- Critical Materials: Strategies for Achieving Sustainability

Manuscripts Due: August 1, 2014

\section{December 2014}

\section{Theme: Materials for Extreme Environments}

- High-Temperature Coatings for Environmental Protection

- Nuclear Applications of ODS and NFA Alloys

- Long-Term Durability of High-Temperature Alloys

- Long-Term Stability of High-Temperature Materials

- Radiation Effects in Oxide Ceramics and Novel LWR Fuels 


\section{4 advisors and committees}

\begin{tabular}{|c|c|c|}
\hline Alexis Lewis & Cong Wang & Yunzhi Wang, advisor; \\
\hline $\begin{array}{c}\text { Advanced Characterization, Testing, } \\
\text { and Simulation Committee }\end{array}$ & Energy Committee & $\begin{array}{l}\text { Paul Gibbs, guest editor; } \\
\text { Seth Imhoff, guest editor }\end{array}$ \\
\hline & Xiaochuan Lu & Phase Transformations \\
\hline $\begin{array}{c}\text { Michael Gao and Sinn-wen Chen } \\
\text { Alloy Phases Committee }\end{array}$ & $\begin{array}{c}\text { Energy Conversion and Storage } \\
\text { Committee }\end{array}$ & Committee \\
\hline $\begin{array}{l}\text { Alton Tabereaux } \\
\text { Aluminum Committee }\end{array}$ & $\begin{array}{l}\text { Tim Gabb and Chantal Sudbrack } \\
\text { High Temperature Alloys Committee }\end{array}$ & $\begin{array}{c}\text { Fernand Marquis } \\
\text { Powder Materials Committee }\end{array}$ \\
\hline $\begin{array}{c}\text { John Nychka and Jaime Kruzic } \\
\text { Biomaterials Committee }\end{array}$ & $\begin{array}{c}\text { Harald Oosterhof } \\
\text { Hydrometallurgy \& Electrometallurgy } \\
\text { Committee }\end{array}$ & $\begin{array}{c}\text { Lifeng Zhang } \\
\text { Process Technology \& Modeling } \\
\text { Committee }\end{array}$ \\
\hline $\begin{array}{c}\text { Mark Asta } \\
\text { Chemistry and Physics of Materials } \\
\text { Committee }\end{array}$ & $\begin{array}{l}\text { Nitin Chopra } \\
I C M E \text { Committee }\end{array}$ & $\begin{array}{c}\text { Phil Mackey, Zhiwei Peng, and } \\
\text { Dean Gregurek } \\
\text { Pyrometallurgy Committee }\end{array}$ \\
\hline $\begin{array}{c}\text { Tomoko Sano } \\
\text { Composite Materials Committee }\end{array}$ & $\begin{array}{l}\text { Alok Singh and James Saal } \\
\text { Magnesium Committee }\end{array}$ & $\begin{array}{l}\text { Gabrielle Gaustad } \\
\text { Recycling \& Environmental } \\
\text { Technologies Committee }\end{array}$ \\
\hline $\begin{array}{c}\text { Nikhil Gupta } \\
\text { Composite Materials Committee }\end{array}$ & $\begin{array}{c}\text { Thomas Woodcock } \\
\text { Magnetic Materials Committee }\end{array}$ & $\begin{array}{c}\text { Todd Leonhardt } \\
\text { Refractory Metals Committee }\end{array}$ \\
\hline $\begin{array}{l}\text { Jonathan Zimmerman, } \\
\text { Adrian Sabau, Mohsen Asle Zaeem, } \\
\text { Mark Tschopp, and Douglas Spearot } \\
\text { Computational Materials Science and }\end{array}$ & $\begin{array}{c}\text { Shadia Jamil Ikhmayies } \\
\text { Materials Characterization } \\
\text { Committee }\end{array}$ & $\begin{array}{c}\text { John Carsley } \\
\text { Shaping \& Forming Committee }\end{array}$ \\
\hline Engineering Committee & $\begin{array}{c}\text { Brad Boyce, advisor; } \\
\text { Saryu Fensin, guest editor }\end{array}$ & $\begin{array}{c}\text { André Phillion } \\
\text { Solidification Committee }\end{array}$ \\
\hline $\begin{array}{c}\text { Computational Materials Science and } \\
\text { Engineering Committee }\end{array}$ & Committee & $\begin{array}{c}\text { Sandip Harimkar } \\
\text { Surface Engineering Committee }\end{array}$ \\
\hline $\begin{array}{l}\text { Raul B. Rebak and } \\
\text { Vilupanur A. Ravi }\end{array}$ & $\begin{array}{l}\text { Terry Xu and Jung-Kun Lee } \\
\text { Nanomaterials Committee }\end{array}$ & $\begin{array}{c}\text { Roger Narayan } \\
\text { Thin Films \& Interfaces }\end{array}$ \\
\hline $\begin{array}{c}\text { Corrosion \& Environmental Effects } \\
\text { Committee }\end{array}$ & $\begin{array}{c}\text { Jian Wang } \\
\text { Nanomechanical Materials Behavior } \\
\text { Committee }\end{array}$ & Committee \\
\hline $\begin{array}{c}\text { Babak Arfaei } \\
\text { Electronic Packaging \& } \\
\text { Interconnection Materials Committee }\end{array}$ & $\begin{array}{c}\text { Raul Rebak } \\
\text { Nuclear Materials Committee }\end{array}$ & Titanium Committee \\
\hline
\end{tabular}

Anyone wishing to publish in JOM should follow the guidelines established in the JOM Instructions for Authors. This material features detailed information on communication, manuscript preparation, and publication procedures. The Instructions for Authors are available on the JOM website at www.tms.org.

For More Information Contact:

Telephone: (724) 776-9000 ext. 228

e-mail: jom@tms.org 